\title{
CRACK DETECTION OF IMMERSED METALLIC STRUCTURE IN WATER WITH SURFACE OSCILLATION USING SCANNING LASER PULSE
}

\author{
J.Y. PARK ${ }^{1}$, J.R. $\mathrm{LEE}^{1}$, B.Y. KOO ${ }^{2}$ \\ ${ }^{1}$ Department of Aerospace Engineering, Korea Advanced Institute of Science and Technology, \\ Daejeon 305-338, Korea. \\ ${ }^{2}$ American Bureau of Shipping, KETC, Busan, Korea.
}

\begin{abstract}
There are some difficulties of non-destructive test and evaluation for immersed or submerged structures such as nuclear reactor pipe line, submarine or huge ship. This paper proposes the method of damage detection of immersed metallic structure which has crack on weld zone and placed in the water with slight and random surface oscillation using ultrasonic wave propagation imaging (UWPI) system with piezoelectric transducer. A T-shape metallic structure with artificial surface crack on weld zone, which with size $2 \mathrm{~mm}$ by $0.3 \mathrm{~mm}$ and the depth $2 \mathrm{~mm}$, used as the specimen. A $532 \mathrm{~nm}$ Q-switched continuous wave laser is used for scanning an area of $20 \mathrm{~mm}$ by $40 \mathrm{~mm}$. A piezoelectric sensor with magnetic sensor head, which is attachable to metallic structure is used as a contact ultrasonic sensor. The tests are performed in three cases: a specimen without water, a specimen immersed in water and a specimen immersed in water with random surface oscillation. Ultrasonic wave propagation image algorithm and adjacent wave subtraction (AWS) algorithm are used for visualizing wave propagation and detecting the crack. For the case where the specimen is immersed in the water, the signal amplitude is increased compared with the specimen without water case. In AWS algorithm results of the immersed case, scattering waves which are generated by cracks were observed. In random surface oscillation case, the excitation laser beam is refracted randomly by Snell's law resulting in the diluted wave propagation images. However, improvements in signal-to-noise ratio using repeat scan technology enable to the detection of the crack and estimation of the crack size.

This paper examines the damage detection using ultrasonic wave propagation caused by laser excitation from outside of the water even when the water surface oscillation exists and implies the possibility of application of ultrasonic propagation imaging system to submerged structures.

Keywords: crack damage, laser pulse scanning, repeated scanning technique, structure under oscillating water, ultrasonic propagation imaging system.
\end{abstract}

\section{INTRODUCTION}

Over the past few decades, a lot of studies related to underwater structures such as nuclear reactor pipe lines, submarines or huge ships have been done. To reduce the cost and time for maintenance, nondestructive testing of the submerged structures during an operation is necessary. Many studies utilize ultrasound for nondestructive testing of submerged structures because ultrasound has very short wavelength; it can be reflected and scattered by very small defects. $\mathrm{Na}$ and Kundu [1] generated flexural cylindrical guided waves on underwater pipes for inspection using a novel transducer holder. Chen et al. [2] proposed corrosion damage identification approach which is capitalizing on the fundamental anti-symmetric mode of Lamb wave in submerged structures. Rizzo et al. [3] presented 
non-contact damage inspection for immersed structures using hybrid laser/immersion transducer system. Sharma and Mukherjee [4] proposed non-contact, in situ and nondestructive monitoring system for corrosion damage detection of submerged plates using a pair of transducers arranged in pitch catch.

When the laser pulse reaches to structure, high energy of laser pulse is transmitted to the local area of the structure. This transmitted energy makes thermoelastic effect where the temperature changes induce to generate elastic wave. There are four primary elastic wave modes: longitudinal wave, shear wave, surface (Rayleigh) wave and plate (Lamb) wave. These waves are different types of ultrasonic wave and are useful for integrated structural health monitoring and nondestructive testing. Chong and Lee [5] developed laser ultrasonic propagation imaging system with twenty-kilohertz scanning frequency using the principle above. In ultrasonic propagation imaging system, various signal processing algorithms are utilized to collect proper information from the signal. Lee and Sunuwar [6] presented various damage visualization algorithms of ultrasonic propagation imaging system. Among them, ultrasonic wave propagation imaging (UWPI) algorithm is simple but effective damage visualization process. Lee et al. [7] and Chia et al. [8] developed adjacent wave subtraction algorithm and applied to the detection of actual damages in composite wing. Using adjacent wave subtraction (AWS) algorithm, anomalous wave caused by damage and so on is effectively observed making it useful for damage detection. To improve the ultrasonic wave propagation images and get a better signal-to-noise ratio, Dhital et al. [9] applied repeat scanning technique to an ultrasonic propagation imaging system and verified that the signalto-noise ratio increases as the number of scanning averaging increases.

In this paper, ultrasonic propagation imaging system, which receive the signal through piezoelectric sensor instead of laser Doppler vibrometer sensor, is used for detecting $2 \mathrm{~mm}$ crack on the weld zone of T-shape metallic structure. To verify the reliability of application of ultrasonic propagation imaging system to submerged specimen, the tests are performed with the specimen placed under randomly oscillating water.

\section{INSPECTION OF SUBMERGED STRUCTURE USING LASER PULSE}

Lee et al. [10] visualized ultrasonic wave propagation and detected the crack damage of liquid-immerse structures using ultrasonic propagation imaging system which are consist of laser pulse excitation to generate Lamb wave on aluminum plate and laser Doppler Vibrometer sensor to signal. According to Lee et al. [10], the depth of water doesn't have affect to wave propagation signal and the system measure the crack size successfully. The size of crack, however, is affected by the density of the liquid. For a water-immersed plate and a glycerin-immersed plate, the crack size is decreased. This result can be explained by Snell's law - the excitation laser beam is refracted when the beam reaches the surface of the liquid.

If the liquid surface is oscillated, the excitation laser pulse will be unstable so that it is impossible to reach to an exact grid point in the scanning area. This lead to the difficulty in the damage detection and the size measurement. For the oscillated water surface case, the difference between original grid point and changed point can be expressed as the eqn (1) below using subscription in Fig. 1.

$$
\mathrm{d}=\mathrm{h} \tan \theta_{2}-\frac{n_{1}}{n_{2}} \sin \left(\theta_{1}-\alpha\right)
$$

However if we magnify the Fig. 1b, laser beam makes not just a point but some circular area as shown in Fig. 2a. Therefore, when the scanning process is repeated, the overlapped 


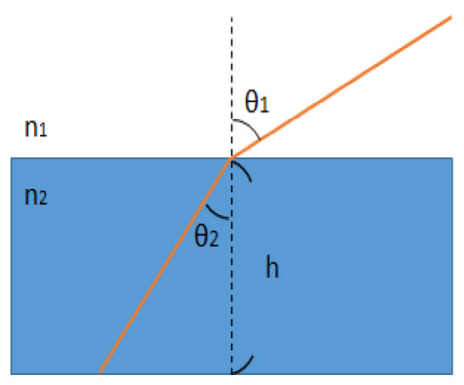

(a)

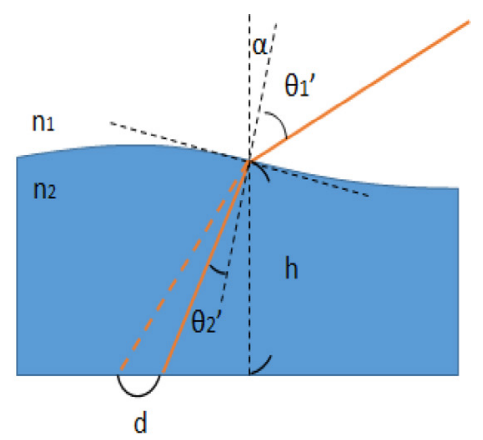

(b)

Figure 1: Snell's Law (a) still surface and (b) oscillating surface.

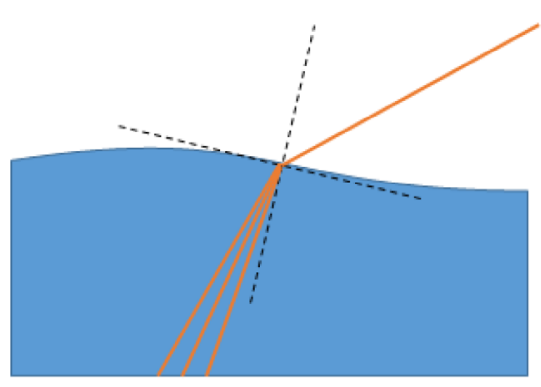

123

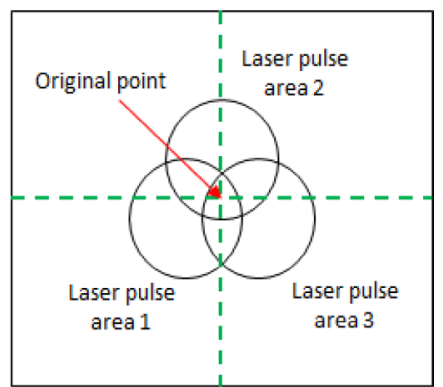

(b)

Figure 2: (a) Repeated laser pulse and (b) overlapped area.

area of the laser beam will close to the original grid when the oscillation is small enough. To verify this, ultrasonic propagation imaging system is utilized for three cases: free specimen, submerged specimen without surface oscillation and submerged specimen with surface oscillation.

\section{EXPERIMENTAL SETUP}

A photo and schematic of the ultrasonic propagation imaging system for submerged structural inspection used in the experiments are shown in Fig. 3a and b. The system consists of in-line signal conditioner with filters and amplifiers, a personal computer (PC) with data acquisition (DAQ) and signal processing algorithms and a $532 \mathrm{~nm}$ Q-switched continuous wave laser. The laser beam with energy of $1.2 \mathrm{~mJ}$, generated by Q-switching technique, is directed to a laser mirror scanner (LMS) so that the laser beam can be reached to the specimen. The scanning area and the interval of laser pulse are $20 \mathrm{~mm}$ by $40 \mathrm{~mm}$ and $0.1 \mathrm{~mm}$, respectively, and the pulse repetition frequency is set to $100 \mathrm{~Hz}$. The ultrasonic wave is measured by a piezoelectric sensor with attachable magnetic sensor head and band-pass filtered between $350 \mathrm{kHz}$ and $500 \mathrm{kHz}$ then stored in the PC. The stored data is processed using algorithms which visualize the wave propagation and the damage, such as UWPI algorithm and AWS algorithm. 


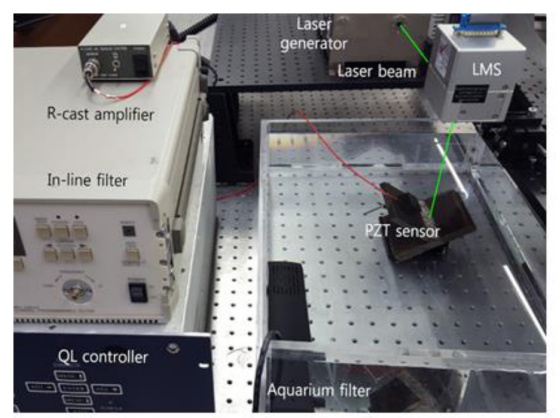

(a)

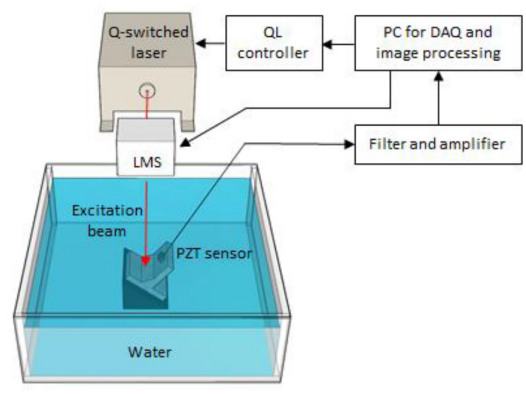

(b)

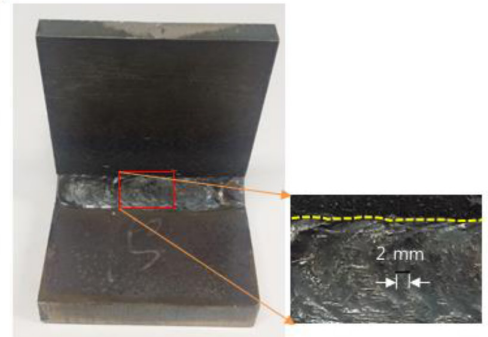

(c)

Figure 3: Experimental Setup (a) photo, (b) schematic of the ultrasonic propagation imaging system for submerged structural inspection and (c) T shape metallic specimen with a $2 \mathrm{~mm}$ crack on weld zone.

The specimen is T-shaped steel material which has the $2 \mathrm{~mm}$ by $0.3 \mathrm{~mm}$ crack is in the middle of the weld joint, as shown in Fig. 3c. Each the thickness of a bottom and a horizontal directional plate of the T-shaped specimen is $15 \mathrm{~mm}$ and $10 \mathrm{~mm}$, respectively. In the experiments, the specimen is located in the middle of the water tank and fixed by an inclined bracket. As mentioned above, the tests are done in three cases: free specimen, submerged specimen without surface oscillation and submerged specimen with surface oscillation. Especially in a submerged specimen case with oscillated water, inspection is repeated 10 times to increase signal-to-noise ratio by repeating scanning technique. An aquarium filter (Hyubshin Water Design Co., LTD.) is located on the water tank corner to make random oscillation of water surface. The hole of an aquarium filter which makes the stream is located $40 \mathrm{~mm}$ above the bottom. Fig. 4 shows the random water surface oscillation caused by the stream.

\section{RESULTS AND DISCUSSION}

\subsection{Ultrasonic wave propagation image results}

The ultrasonic wave is measured by piezoelectric sensor and the signal is stored in the PC. After the inspection, the signal is processed with UWPI algorithm and the movie of ultrasonic wave propagation is made in real time. If the crack exists, the waveform is changed and the scattered or reflected wave is observed. As shown in Fig. 5a and 5b, the crack is detected successfully for the free specimen case and the submerged specimen without any water oscillation case. In the images of ultrasonic wave propagation, scale length is presented so that it 


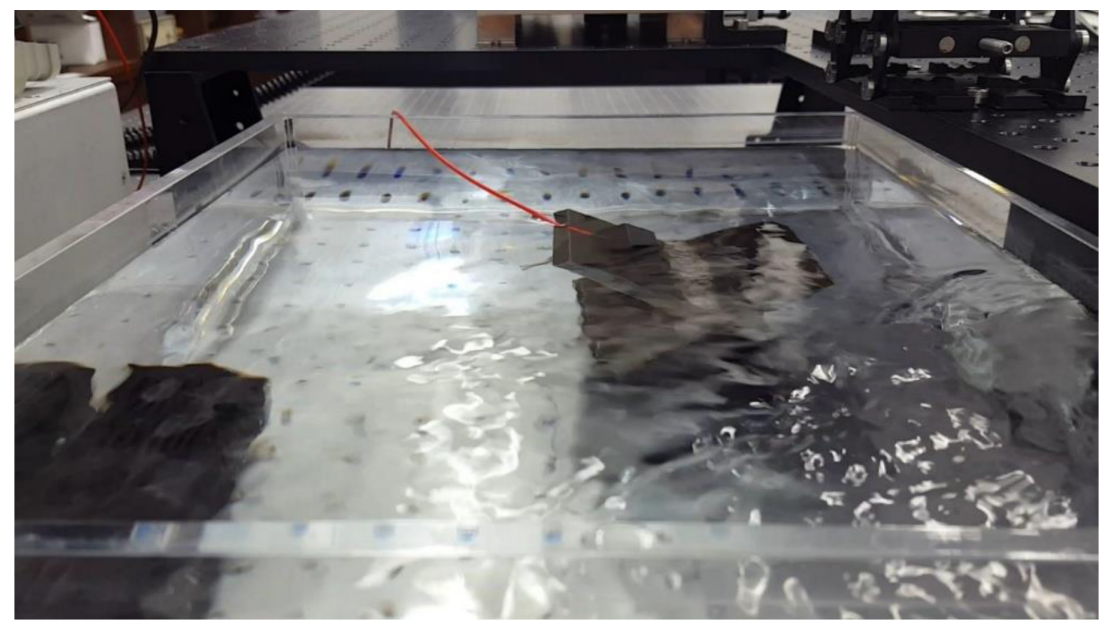

Figure 4: Random water oscillation caused by an aquarium filter.

is easy to estimate the crack size. The measured crack size in Fig. 5a and b is approximately $2 \mathrm{~mm}$ and $2.5 \mathrm{~mm}$. The crack size is increased and the observed location is changed in submerged specimen case because the refracted laser beam is changed its interval length between laser pulses. Also, the signal with large amplitude is observed when the specimen is submerged. It's because the water roles as couplant for piezoelectric sensor so that the amplitude of the measured signal is increased.

For submerged specimen with water oscillation case, the crack is not detected very clearly and difficult to measure the size of the crack. The movie of wave propagation is diluted because the interval length of the laser pulse is irregular and affects to signal processing and the results. It's because irregular laser pulse intervals become regular when the repeated results are overlapped to get an average. The measured crack size of the submerged specimen with the water oscillation case is approximately $2.4 \mathrm{~mm}$. Then compared with the case with the submerged specimen without water oscillation, the results are similar in size and location as shown in Fig. 5b and c.

\subsection{Adjacent wave subtraction image results}

Using the AWS algorithm, the crack damage can be easily detected because the scattering wave or reflected wave is observed clearly in AWS algorithm with 0 degree direction as shown in Fig. 6. In accordance with the results of ultrasonic wave propagation image, for both cases where the specimen is submerged in the water with or without surface oscillation, the measured crack size was bigger than the case where the specimen is outside of the water and the location also changed. For the result of submerged specimen with surface oscillation case, the AWS image is not as clear as that of the without surface oscillation case but the crack detection and the measurements are still possible. The crack is successfully detected in both ultrasonic wave propagation image algorithm and AWS algorithm showing the possibility of the ultrasonic propagation imaging system with repetitive scanning technique application for submerged structures even if the water surface is oscillated randomly. 


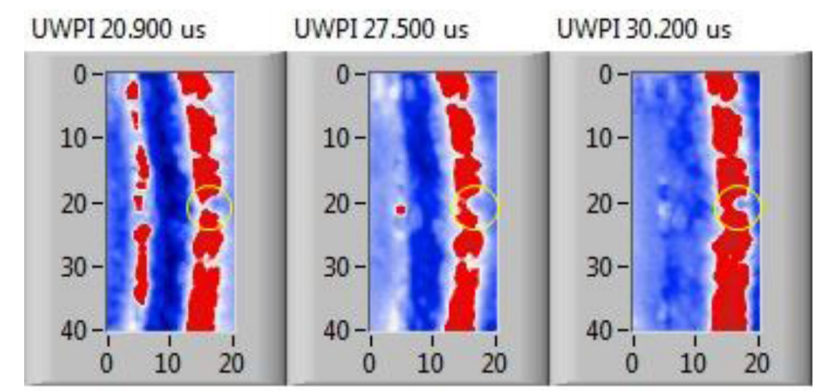

(a)

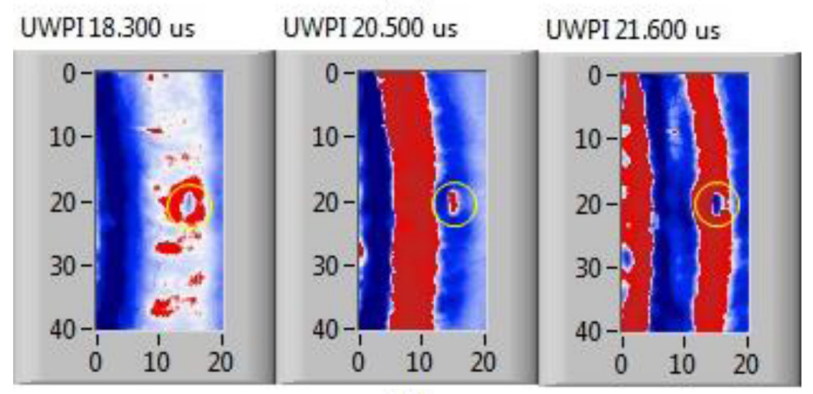

(b)

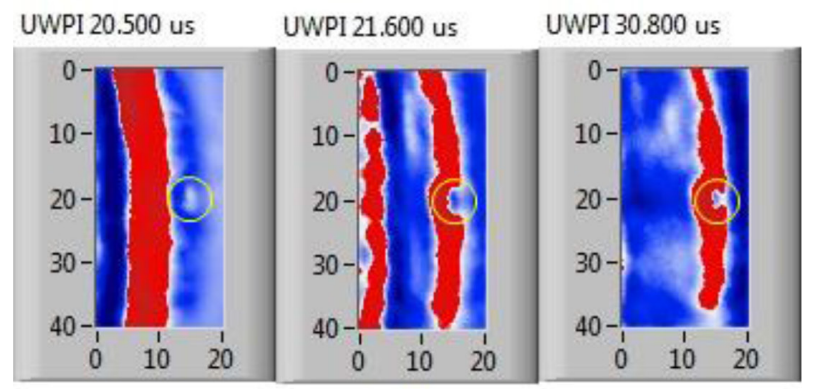

(c)

Figure 5: Ultrasonic wave propagation snapshot images, (a) free specimen, (b) submerged specimen without surface oscillation and (c) submerged specimen with surface oscillation.

\section{CONCLUSION}

When the laser pulse impacts solid structure, ultrasonic wave is generated by thermoelastic effect and damages can be detected. Using this characteristic, ultrasonic propagation imaging system uses $532 \mathrm{~nm}$ Q-switched continuous wave laser to generate ultrasonic waves and visualizes the wave propagation to detect the damage with various signal processing algorithms. When the laser pulse passes through the water surface, it gets refracted affecting ultrasonic wave propagation images of immersed structure. This problem can be solved by the repeated scanning method which enables ultrasonic propagation imaging system to improve the signal-to-noise ratio and the visibility of images, leading to more precise crack detection. To verify the feasibility of ultrasonic propagation imaging system to immersed 


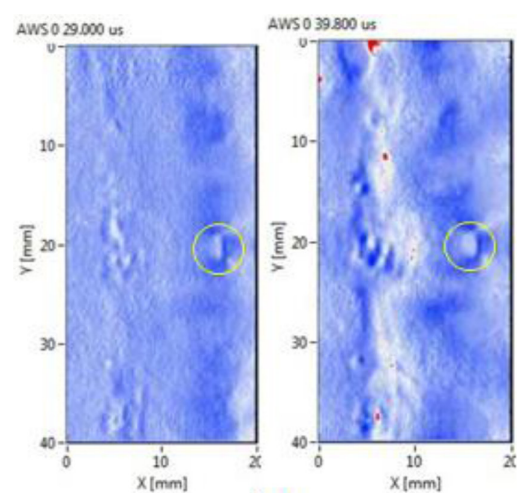

(a)

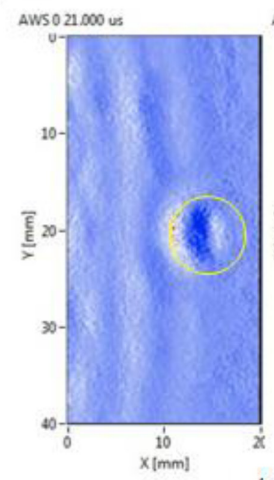

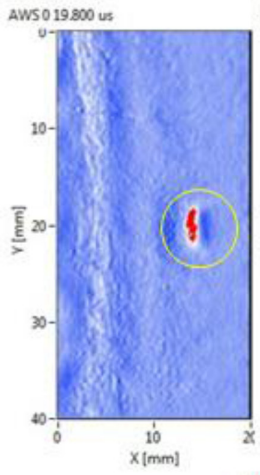

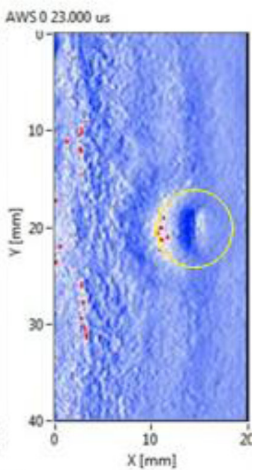

(b)

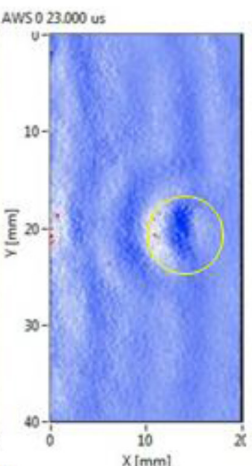

(c)

Figure 6: AWS algorithm results snapshot images, (a) free specimen, (b) submerged specimen without surface oscillation and (c) submerged specimen with surface oscillation.

structure, ultrasonic propagation imaging system is used to examine the T-shape metallic structure which has $2 \mathrm{~mm}$ crack on the weld zone in three cases: free specimen, submerged specimen without surface oscillation, and submerged specimen with surface oscillation. The scanning area was $20 \mathrm{~mm}$ by $40 \mathrm{~mm}$ including crack damage and the inspection was repeated 10 times in submerged specimen with surface oscillation case. In the ultrasonic wave propagation image results and AWS results, ultrasonic propagation imaging system successfully detected the crack even in the case where the random water surface oscillation existed. Especially in AWS results, the scattered wave was observed due to the presence of the crack. The measured sizes of the crack in submerged cases, however, are bigger than that of the free specimen case and the locations of the crack are also slightly shifted. In the free specimen case, the measured crack size was $1.9 \mathrm{~mm}$; otherwise, the measured crack sizes were $2.5 \mathrm{~mm}$ and $2.4 \mathrm{~mm}$ in submerged specimen without and with surface oscillation cases respectively. This result implies the possibility of application of ultrasonic propagation imaging system to submerged structures such as nuclear reactor pipe line, submarine or huge ship.

\section{ACKNOWLEDGEMENT}

This research was supported by Leading Foreign Research Institute Recruitment Program (2011-0030065) and Development of the Composite Lattice Structure (15-CM-MA-12) 
through the National Research Foundation of Korea funded by the Ministry of Science, ICT and Future Planning.

\section{REFERENCES}

[1] Na, W.B. \& Kundu, T., Underwater pipeline inspection using guided waves. Journal of Pressure Vessel Technology, 124(2), pp. 196-200, 2002. http://dx.doi.org/10.1115/1.1466456

[2] Chen, J., Su, Z. \& Cheng, L., Identification of corrosion damage in submerged structures using fundamental anti-symmetric Lamb waves. Smart Materials and Structures, 19(1), p. 12, 2010.

http://dx.doi.org/10.1088/0964-1726/19/1/015004

[3] Rizzo, P., Han, J.G. \& Ni, X.L., Structural health monitoring of immersed structures by means of guided ultrasonic waves. Journal of Intelligent Material Systems and Structures, 21(14), pp. 1397-1407, 2010. http://dx.doi.org/10.1177/1045389X10384170

[4] Sharma, S. \& Mukherjee, A., Ultrasonic guided waves for monitoring corrosion in submerged plates. Structural Control and Health Monitoring, 22, pp. 19-35, 2015. http://dx.doi.org/10.1002/stc.1657

[5] Chong, S.Y. \& Lee, J.R., Development of laser ultrasonic propagation imaging system with twenty-kilohertz scanning frequency for nondestructive evaluation applications. Proceeding of Advances in Structural Health Management and Composite Structures, Jeonju, Korea, 1, pp. 18-21, 2014.

[6] Lee, J.R. \& Sunuwar, N., Advances in damage visualization algorithm of ultrasonic propagation imaging system. Journal of the Korean Society for Nondestructive Testing, 33(2), pp. 232-240, 2013.

[7] Lee, J.R., Chia, C.C., Park, C.Y. \& Jeong, H., Laser ultrasonic anomalous wave propagation imaging method with adjacent wave subtraction: algorithm. Optics \& Laser Technology, 44, pp. 1507-1515, 2012.

http://dx.doi.org/10.1016/j.optlastec.2011.12.008

[8] Chia, C.C., Lee, J.R., Park, C.Y. \& Jeong, H., Laser ultrasonic anomalous wave propagation imaging method with adjacent wave subtraction: application to actual damages in composite wing. Optics \& Laser Technology, 44, pp. 428-440, 2012.

http://dx.doi.org/10.1016/j.optlastec.2011.08.007

[9] Dhital,D., Lee, J.R. \& Park, C.Y., Fully non-contact laser excitation and reception ultrasonic propagation imaging system with repeat scanning technique. Proceeding of 6th European Workshop on Structural Health Monitoring, pp. 1-8, 2012.

[10] Lee, J.R., Jang, J.K. \& Kong, C.W., Fully noncontact wave propagation imaging in an immersed metallic plate with a crack. Shock and Vibration, 2014, p. 8, 2014.

http://dx.doi.org/10.1155/2014/895693 